\title{
Tangence
}

\section{Bords de mer}

\section{La pensée de la marge chez Michelet}

\section{Paule Petitier}

Numéro 57, mai 1998

Littérateurs atypiques et penseurs irréguliers

URI : https://id.erudit.org/iderudit/025971ar

DOI : https://doi.org/10.7202/025971ar

Aller au sommaire du numéro

Éditeur(s)

Tangence

ISSN

0226-9554 (imprimé)

1710-0305 (numérique)

Découvrir la revue

Citer cet article

Petitier, P. (1998). Bords de mer : la pensée de la marge chez Michelet.

Tangence, (57), 96-110. https://doi.org/10.7202/025971ar

Ce document est protégé par la loi sur le droit d'auteur. L'utilisation des services d'Érudit (y compris la reproduction) est assujettie à sa politique d'utilisation que vous pouvez consulter en ligne.

https://apropos.erudit.org/fr/usagers/politique-dutilisation/
Cet article est diffusé et préservé par Érudit.

Érudit est un consortium interuniversitaire sans but lucratif composé de l’Université de Montréal, l'Université Laval et l'Université du Québec à Montréal. Il a pour mission la promotion et la valorisation de la recherche. https://www.erudit.org/fr/ 


\section{Bords de mer. La pensée de la marge chez Michelet Paule Petitier, Université de Tours}

En mars 1851, le cours de Michelet au Collège de France est suspendu, pour sanctionner l'historien d'avoir subordonné son enseignement à la polémique et provoqué des manifestations hostiles au prince-président. Il est destitué en avril 1852, puis, s'étant abstenu de prêter le serment exigé des fonctionnaires par le nouveau régime, quitte les Archives. Il n'occupe plus alors de fonctions officielles.

\section{Décentrement}

Cette situation nouvelle atteint un homme qui, depuis 1827 , vingt-cinq ans, exerce sa fonction et construit sa pratique dans des lieux non seulement officiels, mais produits de la centralisation. En 1827, il a été nommé professeur à l'École normale, création de la centralisation révolutionnaire; en 1831, chef de la section historique des Archives, dont l'organisation et le statut modernes ont aussi été mis en place par la Révolution; en 1838, il est élu professeur au Collège de France, créé au XvI ${ }^{e}$ siècle par le pouvoir royal, et à l'Académie des sciences morales et politiques, fondée en $1795^{1}$. L'œuvre qu'il écrit alors, prolongeant son enseignement, se nourrissant de ses recherches aux Archives est organiquement liée aux institutions auxquelles il appartient.

Sa pensée est alors organisée en fonction de l'idée de "centre". Celle-ci détermine pour lui la compréhension de la formation de la France, telle qu'il l'expose dans le Tableau de la France: il montre comment la nation s'est constituée par gravita-

1 L'histoire de cette dernière institution est retracée par Sophie-Anne Leterrier, L'institution des sciences morales, Paris, L'Harmattan, 1995. L'article 298 de la Constitution de l'an III montre bien le caractère centralisateur qui a présidé à sa création: "Il y a, pour toute la République, un Institut national chargé de recueillir les découvertes, de perfectionner les arts et les sciences." (cité par S.-A. Leterrier, p. 6). 
tion des différentes provinces qui, se soudant et se rapprochant au fil des siècles, en viennent à créer un noyau central, héritier de leurs différentes qualités, récepteur et redistributeur de leur énergie. Ce n'est pas seulement une explication du "centralisme" à la française, dont les autres historiens du $\mathrm{XIX}^{\mathrm{e}}$ siècle reconnaissent le rôle déterminant. La centralisation devient chez Michelet une modélisation du devenir historique. Il convoque les conclusions de la biologie contemporaine pour établir que l'être supérieur est l'être centralisé; la France est à la tête des nations et de l'histoire universelle parce qu'elle a conduit ce phénomène de centralisation à un degré plus parfait que les autres. Le centre n'est pas un lieu dans le Tableau de la France, il est une "fonction "; à l'inverse des provinces fortement caractérisées par leurs particularismes, le centre n'est pas décrit, il reste abstrait. "Plat", " pâle", gris, le centre est concept, et d'une certaine façon, renvoie à une démarche intellectuelle, celle qui conduit des particularités vers l'abstraction qui saisit, sinon l'essence, du moins la relation :

Le caractère du centre de la France est de ne présenter aucune des originalités provinciales, de participer à toutes et de rester neutre, d'emprunter à chacune tout ce qui n'exclut pas les autres, de former le lien, l'intermédiaire entre toutes, au point que chacune puisse à volonté reconnaître en lui sa parenté avec tout le reste. (Introduction à l'bistoire universelle, $1831^{2}$ )

À cette époque, Michelet est parisien, et se réclame de son origine et de son enfance parisiennes. Sa vie est ancrée dans la capitale, même s'il la quitte assez régulièrement pour des voyages en province ou à l'étranger. Paris est son foyer et son lieu mental. Il ouvre ainsi son premier cours au Collège de France, consacré à l'histoire de Paris:

Je suis né à Paris, j'y ai vécu, j'y serai enterré, s'il plaît à Dieu. Je dois beaucoup à cette ville. Mes deux vies s'y rapportent, tout entières, ma vie de passion, ma vie d'idées, celle de l'homme et celle de l'écrivain. Pour ne parler que de la seconde, c'est à Paris que je dois d'avoir constamment senti le rayonnement de la France. ${ }^{3}$

À partir de 1852, délié de ses fonctions officielles, Michelet entame un autre type d'existence, plus du tout centré sur Paris. La désaffection pour une capitale qui, de symbole de la révolution qu'elle

2 Guures complètes, Paris, Flammarion, 1972, t. II, p. 248.

3 Note inédite du Cours de 1838 (BHVP, A. 3739, fo 52). 
98

était avant 1848 , est devenue le lieu de la brutale répression de juin, de l'absence de résistance populaire au coup d'état de 1851, puis de la fête impériale ${ }^{4}$, est un phénomène assez général. Toujours est-il que pour différentes raisons, politiques, financières, de santé, Michelet et sa jeune femme, partageront leurs années sous le Second Empire entre des séjours dans des lieux de villégiature estivale et des hivers parisiens. Dès juin 1852 , juste après sa destitution du Collège de France et des Archives, l'historien avait décidé de s'installer à Nantes pour terminer l'Histoire de la Révolution et exploiter les archives concernant la guerre de Vendée. Donc, pour achever l'histoire de la Révolution, non seulement il se déplace à la périphérie du territoire, mais l'objet historique qui l'y attire manifeste la volonté d'analyser la fin de la Révolution du point de vue de son échec, des résistances qu'elle a suscitées. II est important que le déplacement vers la marge accompagne une réflexion sur l'impensable de la Révolution, sur la révolte des paysans de l'Ouest, se retournant contre des décisions d'un pouvoir central qui voulait les émanciper, sur la Terreur, transformation de la force de libération en violence dictatoriale.

Au cours des années suivantes, les Michelet, sacrifiant à la mode balnéaire, au "désir du rivage" dont Alain Corbin a étudié l'éclosion ${ }^{5}$, vont séjourner au bord de différentes mers. A Nervi, petit port près de Gệnes, à Granville, sur la Manche, à SaintGeorges-de-Didonne, au bord de la Gironde, à Etretat, SaintValéry-en-Caux, Hyères, Toulon... Le point commun de ces lieux est leur situation périphérique par rapport au territoire français. Leur multiplicité souligne une sorte de nomadisme: les Michelet ne désirent pas se fixer dans un lieu qui serait un substitut de Paris.

Tous ces éléments biographiques n'auraient pas grande importance s'ils ne donnaient lieu à des œuvres. La mer (1860), plus tard La montagne (1868), sont avant tout des livres sur des lieux, des livres dans lesquels la représentation de l'espace est capitale. Or chez Michelet les schémas spatiaux sont indissocia-

4 Dans Le spleen contre l'oubli. Juin 1848 (Paris, Payot, 1996), Dolf Oelher parle de ce dégoût éprouvé pour la capitale après juin tant par ceux qui sont "indignés par un gouvernement trop mou " que par ceux "qui désespèrent de la république et de Paris" (p. 96 et ss.).

5 Alain Corbin, Le territoire du vide. L'Occident et le désir du rivage. $1750-$ 1840, Paris, Aubier, 1988.

6 C'est entre autres ce que j’ai voulu montrer dans La géographie de Michelet, Paris, L'Harmattan, 1997. 
bles de l'exposition du mode de pensée. On pense dans un certain espace, en fonction de sa situation dans celui-ci, et les lois et la structure de cet espace figurent des opérations de pensée. Se représentant comme un exilé de l'intérieur, Michelet écrit des livres sur les marges, les espaces marginaux (plages, landes, massifs montagneux), les comportements marginaux (sorcellerie); des livres qui sont eux-mêmes en marge de sa production d'historien, de son grand cuvre de l'Histoire de France poursuivi en parallèle. Des livres en marge tout court. Ouvrages de vulgarisation mêlant un savoir scientifique de seconde main à des observations tirées d'expériences vécues, se voulant à la fois libre réflexion philosophique, vision du monde et livres pratiques, manuels de vie, d'hygiène, de conduite, ils restent inclassables.

\section{Anamorphose du centre}

L'espace privilégié, matriciel, de la marge, c'est la mer. Et dans l'histoire de l'œuvre, on peut dire que La sorcière (1862), œuvre à laquelle on pense spontanément lorsqu'on songe à l'intérêt de Michelet pour ce qui sort de l'histoire traditionnelle, est fille de La mer (1860), n'aurait pu être écrite si au cours des deux années précédentes Michelet n'avait mis en œuvre une épistémologie de la marge qui s'expérimentait au bord de différentes mers depuis le séjour à Nervi de 1853. Marge bien évidemment la plage, cette lisière disputée où se découvre le monde marin, où se défait la terre. Mais bientôt marge la mer tout entière, la plaine liquide, qui d'abord se donne à voir comme vide, stérilité, deuil clair autour du texte-terre.

L'aventure de la marge sert à déconstruire le point de vue du centre, à en opérer la critique. Dans La mer, Michelet évoque la dangereuse plage qui entoure le Mont Saint-Michel, image même du lieu marginal, vaste lisière blanche sans identité définie, ni terre, ni mer, ni eau douce. Or cette description est faite à partir d'un point de vue:

Au plus haut de Saint-Michel, on vous montre une plate-forme qu'on appelle celle des Fous. Je ne connais aucun lieu plus propre à en faire que cette maison de vertige. Représentez-vous tout autour une grande plaine comme de cendre blanche. (53)

Point de vue non innocent. Extrêmement élevé, il implique une vision surplombante. D'autres caractères le désignent comme vision du centre. Par exemple, le paysage l'entoure. 
100

Le Mont Saint-Michel est décrit plus loin comme un entassement de prisons:

Sur un gros bloc de granit, il se dresse, monte et monte encore indéfiniment, comme une babel d'un titanique entassement, roc sur roc, siècle sur siècle, mais toujours cachot sur cachot. Au plus bas, l'in pace des moines; plus haut, la cage de fer qu'y fit Louis XI; plus haut, celle de Louis XIV; plus haut, la prison d'aujourd'hui. (53-54)

Une telle description donne à comprendre le sens du monument dans ce texte. Il représente ici le pouvoir de l'état, on reconnaît les figures clefs de la monarchie centralisatrice et absolutiste (Louis XI, Loujs XIV). Défini par la verticalité, par la fonction carcérale, il désigne la vision linéaire et mécaniquement progressiste d'une histoire comprise uniquement en fonction de l'État. Sur les marges on voit ainsi l'envers du pouvoir, l'enfer qui assure sa pérennité.

Depuis la première phrase de présentation jusqu'à cette découverte, il s'est produit une inversion de point de vue: on est descendu de la tour d'où l'on observait la grève, on a mis le pied sur la marge maléfique, pris le risque de s'y voir engloutir, échappé au danger et touché au bloc de granit pour le reconsidé$\mathrm{rer}^{7}$. Ce risque encouru - bien peu vraisemblable - doit être lu comme la clef d'un retournement de l'interprétation. Celui qui le court touche le réel de la marge, découvre une nouvelle lecture. En fait, depuis le début le texte désigne le point de vue central comme celui de la folie. C'est bien la tour qui est une "maison de vertige"; c'est bien elle qui produit la folie (Je ne connais aucun lieu plus propre à en faire que cette maison de vertige.). La plage traîtresse n'est rien d'autre que la projection en négatif de la tour, "l'abîme" qui cherche à vous y happer rien d'autre que la verticalité du mont renversée.

La marge, nous dit ce texte, n'est que l'envers de la création du centre; plus puissant sera celui-ci, plus dangereuse elle sera:

Est-ce la faute de la mer si cette plage est perfide? [...] La faute est surtout à l'homme, à son ignorance, à sa négligence. Dans

7 Cf. ibid.: "Et, si l'on passe quand l'eau se retire, on risque d'être englouti. J'en puis parler, je l'ai été presque moi-même. Une voiture fort légère, dans laquelle j'étais, disparut en deux minutes avec le cheval; par miracle, j'échappai. Mais moi-même à pied, j'enfonçais. À chaque pas, je sentais un affreux clapotement, comme un appel de labîme qui me demandait doucement, m'invitait et mattirait, et me prenait par-dessous. " 
les longs âges barbares, pendant qu'il rêve à la légende et fonde le grand pélerinage de l'archange vainqueur du diable, le diable prit possession de cette plaine délaissée. (54)

Un tel commentaire peut être lu à plusieurs niveaux: il fait écho à la critique - classique au $\mathrm{XIX}^{\mathrm{e}}$ siècle - des méfaits de la centralisation: l'énergie mobilisée pour le centre laisse en jachère d'autres espaces. Il s'offre simultanément à un décryptage plus politique. La mer est une métaphore convenue pour dire le peuple; Michelet joule tout au long de l'ouvre sur cette possibilité latente d'encodage. La plage du Mont Saint-Michel montre que l'énergie déployée pour la construction de l'appareil d'État a laissé de côté le peuple, et celui-ci est devenu une plaine dangereuse, apparemment inerte, infiltrée de courants invisibles qui en font cette chose imprévisible, sur laquelle on ne peut pas compter, dans laquelle les républiques peuvent s'engloutir en moins de temps qu'il ne faut pour les construire. Le groupe de l'archange Saint-Michel terrassant le démon, élevé au dessus de la fontaine du même nom à Paris après 1848 , symbolise la victoire de l'ordre sur l'anarchie ${ }^{8}$. Jouant sur ces clichés politiques de l'histoire récente, la réflexion de La sorcière sur l'imaginaire se met déjà en place ici. La marge est le lieu du diable, et le diable n'est que l'envers de Saint-Michel: la tour chavire dans l'imaginaire, elle est du côté de la légende (la légende royale ${ }^{9}$, la légende du pouvoir); la plage aussi se renverse dans le fantastique, devient l'envers noir de la légende dorée. Annexés à l'imaginaire, les deux lieux n'en continuent pas moins d'exister, et n'en perdent pas pour autant leur efficace sur le réel, mais le rapport avec le réel y est perverti, d'une façon qui ne peut que les condamner tous les deux à la stérilité : la tour est au centre d'un tourbillon. Lieu de la vanité (Babel y évoque le péché d'orgueil lié à une volonté d'unité pleine d'hybris), elle est logiquement celui du vent, du "trouble éternel». La plaine quant à elle n'est qu'un désert de cendre blanche.

8 Voir en particulier à ce propos le texte de Castagnary, "la Fontaine SaintMichel ", 15 août 1860, dans Paris qui s'en va et Paris qui vient, Paris, A. Caclart, 1865 .

9 Saint Michel était le patron du royaume de France et de la couronne. 
102

\section{Où le centre n'est pas celui qu'on croit}

La figure du Mont Saint-Michel prend une forte valeur symbolique de ce qu'elle figure dans le lieu même de la marge l'apparition archétypale du centre. On voit que s'y noue une critique de la centralisation comme phénomène politique et comme modèle de pensée.

Il faut pourtant introduire une distinction. Michelet ne met pas en cause la centralisation comme phénomène d'organisation d'un tout par la mise en relation de ses parties; cela reste pour lui la condition d'un progrès. On retrouve dans La mer des apologies de l'être centralisé (l'oursin, le poisson). Il ne faudrait pas confondre un type de centralisation positif - celui qui a créé la nation, celui qu'a mis en ouvre dans un premier temps la Révolution française - et le phénomène de concentration que Michelet voit à l'œuvre en 1793-1794 à travers l'emprise jacobine et la réduction de la Révolution au seul Robespierre. Politiquement, cela correspond à la figure du tyran, dont l'œuvre de Michelet, à partir du Second Empire, ne cessera d'explorer la généalogie. Le tyran asseoit son pouvoir sur une religion, ce qui le caractérise étant l'"idolâtrie politique".

Car le roi moderne, aux temps chrétiens, tout en portant l'épée, a la chappe, le caractère de prêtre. (Bible de l'bumanité, 358)

La tyrannie va donc de pair avec le monothéisme, et avec ce monothéisme particulier, fondé sur l'incarnation de Dieu, qu'est le christianisme.

Géographiquement, l'espace de la tyrannie est celui de l'empire (non de la nation): empire d'Alexandre, empire romain, et au $\mathrm{XIX}^{\mathrm{C}}$ siècle empires anglais et français. Dans La sorcière, Michelet décrit la façon dont l'empire romain a transformé les dieux du paganisme. Ils n'ont été admis dans la capitale qu'au prix d'une castration qui les a privés de leur fécondité pour en faire de simples outils de l'appareil d'État:

Pour les recevoir, il est vrai, Rome avait pratiqué sur eux une sévère opération, les avait énervés, pâlis. Ces grands dieux centralisés étaient devenus, dans leur vie officielle, de tristes fonctionnaires de l'empire romain. (La sorcière, 46)

L'instrument qui opère cette concentration est en effet l'État, institution qui en France se développe en même temps que s'unifie la nation, mais que Michelet refuse depuis le début de son ouvre 
historique d'identifier à la nation. L'église catholique pour Michelet (en particulier dans certaines de ses formes comme les Jésuites) apparaît comme un simple double de l'appareil d'État, une mécanique pour créer des sujets tous semblables et sans liberté de conscience, son dogme n'est en fait pas distinct de celui de tout despotisme, c'est l'amour de l'Un.

La marge ne s'oppose pas au centre en tant que tel, mais au centre perverti par l'Un. Le centre-fonction de la nation s'oppose au centre-fin de l'Empire ou de l'État.

Dans La mer, le poisson incarne l'être parfaitement centralisé grâce à "un puissant système nerveux, à nombreux fils télégraphiques qui vont sonner, retentir à l'épine et au cerveau", "un grand système un, mais très-multiple" (192). Le centre reste ici une pure fonction, vouée à la coordination des différentes parties, son utilité est énergétique, et cela par le biais d'une transmission d'informations, et non par l'exercice d'un pouvoir. Dans le Tableau de la France, déjà, Paris n'était que "l'intermédiaire entre toutes lles provinces]", sa fonction celle d'un sensorium. Cependant à cette date, cette fonction lui conférait une "supériorité" sur les provinces. Sous le Second Empire, il s'agira d'éclaircir cette équivoque: la position centrale ne confère aucune supériorité, l'image du réseau l'emporte d'ailleurs sur celle d'un point central. L'organisation centralisée du poisson est faite d'un "riche écheveau de filets nerveux". En effet, on peut dire que les catastrophes historiques de la France proviennent de ce que le lieu de l'échange a été dénaturé en lieu du pouvoir. Le tyran (Napoléon III par exemple), c'est celui qui s'approprie une position centrale, le point névralgique d'un réseau (le Paris révolutionnaire) pour en faire un insturment de domination (la capitale de l'Empire). Dans ce cas, le centre, naguère pure fonction, s'incarne, se matérialise, la cité devient ville et capitalise les richesses, le pouvoir exécutif homme et confisque les libertés... et la marge naît de ce mouvement d'absorption.

Le mode de pensée lié à la centralisation tyrannique est celui d'une pensée hiérarchisée, selon le modèle de l'abstraction, de la généralisation. Il s'enracine dans la religion judéo-chrétienne fondée sur le dualisme du corps et de l'esprit. Ce dualisme légitime une démarche d'abstraction, qui isole à des niveaux différents le fait et l'idée, la réalité et les qualités ou les relations qui permettent de la comprendre, les choses et le langage. L'ennemi de Michelet, et cela très tôt, c'est "la culture tout abstraite que l'on nous donne, cette quantité de formules qui encombrent, énervent la pensée, 
enfin l'éducation de l'École" (p. 34), cette "philosophie des mots, abstraction d'abstractions" (36). L'éducation moderne reprend en cela le principe de l'éducation des jésuites. Michelet condamne les philosophes d'état, comme Victor Cousin, qui réduisent l'histoire au monde des idées, les historiens d'état, comme Guizot, qui restreignent le gouvernement à celui de l'opinion. La langue de Rousseau, dont il affirme la filiation avec celle des logiciens de Port-Royal, lui paraît cristalliser ce type de pensée abstraite et en donner pour longtemps le modèle à la bourgeoisie. L'induction elle-même, lorsqu'elle conduit à séparer à travers différentes étapes le niveau du concret et celui de la pensée, ne peut convenir à Michelet et une démarche comme celle de Taine et de la science positive lui paraissent tout aussi inacceptables. Que la science isole ce qu'elle étudie; et qu'elle l'abstraie pour en retirer le générique, c'est encore le même mouvement stérilisant vers l'unité. Ce qui est catastrophique ce n'est pas l'abstraction en elle-même, c'est l'abstraction considérée comme fin de la pensée, alors qu'elle ne devrait être qu'un outil pour réactiver la saisie du concret.

Si la centralisation maléfique se fonde sur une séparation du corps et de l'esprit, au bout de son processus elle rétablit cependant une identification perverse des deux. Dans la centralisation positive, l'esprit est comme une énergie (comparée à l'électricité) qui "Spiritualise" constamment la matière en la traversant, lui apportant des informations, la rendant capable d'action. La centralisation négative se caractérise par l'incarnation in fine d'un principe complètement abstrait (la puissance de l'état incarnée dans un homme). Elle est donc autant prépotence du corps que domination de l'esprit. La marge dévoile justement cet aspect. La religion qui enferme les femmes dans des couvents, exalte l'esprit et la mortification de la chair, se renverse en hystérie, dans laquelle la pensée tout entière est aliénée au corps, devient parole errante, inassignable, du corps.

C'est donc toute une vision du monde, liée à un mode de pouvoir, mais aussi à une religion et à un système de pensée, qui se trouve remise en cause. La marge n'est périphérique que dans cette logique de pouvoir. Chez Michelet, elle correspond à trois opérations différentes :

- une opération de commutation: l'épreuve périlleuse de la marge, division du sujet, dislocation des grilles d'interprétation habituelles. La marge oppose au pouvoir un contre-pouvoir tout aussi dangereux. 
- une fonction de critique: la marge fait apparaître la monstruosité du système dans lequel on était jusqu'alors inclus. Au cours de cette opération, la marge déborde progressivement, ses caractères se répandent sur ce dont elle paraissait d'abord l'envers noir pour en révéler la vérité. La sorcellerie est un phénomène marginal qui permet la critique de tout un fonctionnement social, le fantastique de la sorcellerie dénonce une forme d'irréalité qui concerne la société entière. La deuxième partie de $L a$ sorcière remplit cette fonction critique, dénonçant la construction dans les manuels de sorcellerie d'une absurdité en miroir, montrant comment la sorcellerie contamine complètement l'Église; elle dévoile la perversité du cloître et de la direction de conscience et rend visible toute l'irrationalité des siècles de la monarchie absolue.

- la mise en place d'un nouveau mode de pensée: la marge est aussi un lieu de savoir, offrant la possibilité de repenser la totalité selon les formes vivantes. La sorcellerie, avant de se dénaturer caricaturalement aux siècles monarchiques, correspond pour Michelet à l'invention des sciences de la nature, aux retrouvailles avec la nature et le réel. Le savoir de la plage s'étend à la mer à partir de laquelle on peut reconstituer une lecture alternative du monde et de la création.

\section{Pensée de la marge}

La marge est l'espace de la planéité, la vaste étendue des grèves (que Michelet préfère aux falaises), la mer (dont la profondeur joue un rôle très minime dans La mer), les marches de l'Ouest, des "landes à perte de vue"; plus tard, cela pourra être la montagne, car l'horizontalité du paysage n'est pas essentielle, elle asseoit dans un premier temps un mode de relation, un régime du sens. Face à la pensée hiérarchique, la marge va arrimer la production du sens à des opérations intellectuelles fondées sur le refus de toute hiérarchisation de la pensée.

À la verticalité de la première vision du Mont Saint-Michel et de l'abîme de sables mouvants qui l'enserre, fait pendant une deuxième lecture du paysage:

Loin de faire mal, au contraire, elle apporte, cette furieuse dans ses flots si menaçants, un trésor de sel fécond, meilleur que le 
106

limon du Nil, qui enrichit toute culture et fait la charmante beauté des anciens marais de Dol, de nos jours transformés en jardins. C'est une mère un peu violente, mais enfin, c'est une mère. Riche en poissons, elle entasse sur Cancale qui est en face, et sur d'autres bancs encore, des millions, des milliards d'huitres, et de leurs coquilles brisées elle donne cette riche vie qui se change en herbe, en fruits, et couvre les prairies de fleurs.

Replacé dans l'espace de la carte (Dol, Cancale), le paysage convertit la dimension historique en espace: l'évolution séculaire du tracé du rivage met en évidence trois lieux contigus, mer, marais, jardins. La mise en espace de l'histoire est essentielle pour contrebalancer sa tendance à organiser toute la compréhension selon l'axe linéaire de l'irréversibilité (qui se prête à toutes les philosophies de la fin de l'histoire). Déployée dans l'espace, l'histoire devient le lieu de la pluralité et de la fécondité. L'entassement des coquilles sur le rivage, gage de fertilité païenne (le limon du Nil), répond à l'entassement des prisons sur le Mont Saint-Michel, image de la stérilité monothéiste. La pensée de la marge est celle qui refuse de subsumer la multiplicité concrète dans le générique. À l'intelligibilité de l'abstraction, elle oppose le principe de compréhension de la métamorphose, qui rend compte du passage d'une forme concrète à une autre, de la transformation des huîtres en fleurs.

La mer donne pourtant au livre II, "La genèse de la mer", une représentation de la chaîne des êtres. Treize chapitres suivent du mucus marin, gelée de vie mêlée à l'eau, jusqu'aux mammifères amphibies - le développement des formes de vie marines. Les différentes espèces évoquées successivement (les coraux, les méduses, l'oursin, les crustacés, le poisson...) ne construisent cependant pas les degrés ascendants d'une évolution linéaire. Chacun est une sorte d'essai, l'expérience d'un être au monde selon une modalité particulière, mais sans postérité. L'énergie créatrice (dont la forme matérielle est cette mucosité propre à l'eau de mer) expérimente et semble repartir à chaque fois de zéro; les formes se résorbent en elle pour une création nouvelle. C'est d'ailleurs par la découverte de cette résorption que commence la série. Le début du chapitre "L’atome" décrit la décomposition de trois créatures oubliées dans un seau, un oursin, une étoile de mer et une ophiure. "Une pellicule épaisse et gélatineuse s'était formée à la surface. "(124) Un atome de cette substance placé sous le microscope révèle "une fermentation vivante" 
(125), un fourmillement de vibrions; "un tourbillon d'animaux" (124). L'évolution n'est pas à sens unique, les créatures supérieures en se décomposant reconstituent le mucus primitif qui de nouveau donne naissance à des formes de vie. Michelet dans $L a$ mer fait siennes les théories de Félix Pouchet, l'adversaire de Pasteur, sur la génération spontanée. C'est qu'elles sont cohérentes avec un mode de pensée non généalogique.

La description michelettiste refuse aussi le principe hiérarchique et centré de la perspective: le microscope sert surtout dans les expériences de Michelet à l'écrasement de l'échelle des proportions, il révèle le gigantisme non relatif mais réel du monde invisible. Le microscope sert à découvrir que les infimes sont des "faiseurs de monde" et que montagnes et continents se constituent de leurs dépouilles. L'infiniment petit devient le démesurément grand. Michelet est par ailleurs d'accord avec Pouchet et Ehrenberg pour penser que ces animalcules révélés par le microscope sont "compliqués, très-élevés d'organisation" et leur prêter "cent vingt estomacs". Un des principes de hiérarchisation de la chaîne des êtres était la loi de complexification croissante des organismes. À chacun de ces êtres, le texte michelettiste prête la parole, chacun devient à son tour l'énonciateur d'un discours sur lui-même, le flux de la parole circule parallèlement à celui de la vie. Chaque spécification permet à un sujet de se constituer, et en tant que sujet, de se poser comme l'équivalent, indépendamment de toute proportion, de tout autre sujet:

Que serait-ce si le rotifère pouvait concevoir l'être collectif où
sommeille un infini, par exemple, la superbe, la colossale
éponge étoilée que vous voyez au Muséum? Elle est à lui ce
qu'est à l'homme le globe même de la terre avec ses neuf mille
lieues de tour. Eh bien, je suis convaincu que dans cette com-
paraison, loin d'en être humilié, l'atome aurait un accès d'or-
gueil et dirait: "Je suis grand." (141)

Aussi la chaîne des êtres de la mer n'est-elle pas finalisée, et Michelet n'envisage pas de continuité entre les races de la mer et la vie terrestre. Le livre tend clairement à suggérer une alternative entre deux façons de comprendre la création. La mer ne tend qu'à produire la mère, la série de ses productions s'achève avec les amphibies à qui leur morphologie permet enfin d'allaiter leur petit et de les serrer contre eux. "La nature s'ingénie dans l'idée fixe de caresser l'enfant, de le prendre et de l'approcher. "(214) Cette dernière transformation morphologique rend possible 
108

l'éducation et le progrès qui chez Michelet ne peuvent fonctionner que selon une réciprocité où l'éduqué devient l'éducateur de son éducateur ${ }^{10}$.

Les amphibies nous ont ramenés sur la marge; et sur la plage, les deux séries, marine et terrestre, vont se trouver confrontées. La série terrestre ne peut se dire qu'en termes hiérarchiques, elle n'apparaît qu'à travers son "couronnement", "le fort des forts, l'ingénieux, l'actif, le cruel roi du monde" (217). L'homme tient ici le rôle du tyran; il réintroduit la logique de l'Un. Sur la plage, où l'on retrouve l'univers du pouvoir, sa logique, son mode de pensée, l'autre mode de pensée se défait et se renverse dans le fantastique (signe de la présence d'un point de vue aliénant). Les amphibies sont des "sirènes" (titre du chapitre): c'est qu'ils font illusion à distance, évoquent des "ombres de la femme". Sans doute y eut-il de vraies sirènes, pense Michelet, mais elles ont été exterminées par les hommes qui les considérèrent comme des monstres. L'humanisation des peuples de la mer, effective depuis l'atome à travers les performances langagières de chaque créature marine, cesse dès que l'on atteint la plage où se manifeste la domination de l'homme, tritons et sirènes "seraient restés muets, dans l'impuissance de se faire un langage" (214). La plage manifeste l'incompatibilité des deux modes de pensée et l'utopie que représente le monde de la mer: l'homme que tendait à créer cette démocratie de l'horizontalité reste une chimère; tant que régnera la logique tyrannique de l'Un, les républiques seront sacrifiées dans les marges de l'histoire

\section{Mobilis in mobile}

Le mode de pensée qui s'expérimente dans les œuvres d'histoire naturelle de Michelet, refusant les structures hiérarchiques et généalogiques s'apparente à ce que Deleuze et Guattari ont désigné par la métaphore du "rhizome"11. Dans La mer, l'énergie vitale est rhizomatique; à l'image du Gulf Stream et du Kuro Shivo, ces "deux voies lactées de la mer", "matrice[s] profonde[s] d'un monde d'êtres vivants" (68), elle laisse se stabiliser d'endroits en endroits une espèce. Pour la saisir, la pensée privilégie

10 Voir Jacques Rancière, "La fleur pétrifiée", dans Courts voyages au pays du peuple, Paris, Seuil, 1990, p. 91-111.

11 Giles Deleuze et François Guattari, Rbizome, Paris, Minuit, 1976. 
les communications transversales. Michel Serres a montré ${ }^{12}$ que l'analyse du dispositif spatial de La mer pouvait se faire selon les différentes régions de l'encyclopédie: géométrie, mécanique, thermodynamique, chimie, biologie, etc. La particularité de ce discours est en effet de procéder à une supersposition continue des significations. En n'importe laquelle de ses descriptions, le texte, nous l'avons vu à propos du Mont Saint-Michel, peut se lire comme une analyse géographique ou topologique, comme un discours sur la politique parallèle d'une réflexion sur la religion et l'imaginaire, comme un métadiscours épistémologique sur son mode de pensée ou sur celui qu'il refuse. Cette polysémie particulière pose le caractère indissociable des différents niveaux d'interprétation: il ne se construisent pas selon un mouvement ascendant, s'éloignant de plus en plus du concret. L'abstraction existe dans le mouvement de l'interprétation, mais ne peut s'autonomiser de la signification concrète dite dans les mêmes mots.

Le monde marin est pour Michelet un objet de savoir, mais il est tout autant une métaphore d'un mode de pensée. La pensée non hiérarchique, non centralisée, est une pensée de la métamorphose et de la fluidité, dont la devise pourrait être celle du capitaine Nemo, "mobilis in mobile", et l'emblème le poisson, mouvement fait chair ${ }^{13}$. La démarche de connaissance se caractérise chez Michelet par une fluidité qui repose sur la mise en communication des différents domaines intellectuels et scientifiques, et qui suppose aussi une évolution et une réévaluation constantes dans le temps de ses procédés et de ses résultats. Il serait contraire à la nature même de cette démarche de se fixer en une "méthode", en un "exposé des principes". Cela reviendrait à ce que récuse Michelet: abstraire et figer les outils intellectuels de la connaissance et fixer une intelligibilité dont le caractère historique consiste justement en cette capacité d'autotransformation. L'œuvre de Michelet pratique donc une réflexivité continue, et invite à se lire à chaque moment comme un métadiscours métaphorique. Dans cette perspective, la marge constitue un des lieux privilégiés de réflexion, où l'épistémologie de l'historien et celle qu'il refuse se renvoient leur image.

12 Michel Serres, "Michelet, la soupe", dans Revue d'bistoire littéraire de la France, septembre-octobre 1974, p. 787-802.

13 Dans les ÉClaircissements de La sorcière, c'est à un poisson que se compare l'historien dans sa démarche d'exploration de la sorcellerie (voir p. 289). 
110

La modalité cognitive indéterminée qui caractérise l'œuvre de Michelet explique en partie la marginalisation qu'il a subie en tant qu'historien dès le Second Empire, et peut-être celle dont il est victime aujourd'hui en tant qu'auteur. Sous le Second Empire, à un moment où l'histoire tend à évoluer vers les exigences du positivisme, la démarche de Michelet paraît trop dépourvue des critères contemporains de la scientificité; refusant d'expliciter clairement sa méthode, elle apparait comme en étant dépourvue. Sa méthode propre, intégrée, suscitant la multiplicité du sens selon des procédés métaphoriques, contribue au contraire à le faire ranger par la critique du côté de la littérature, de la poésie. Aujourd'hui, lorsqu'il n'est pas réduit à sa figure institutionnelle, qui peut intéresser pour son rôle dans l'histoire de la discipline historique, Michelet dissuade sans doute les analystes litterraires parce qu'ils sentent bien que son écriture - aspect essentiel de son œuvre - ne relève pas d'un projet littéraire mais scientifique. 